\title{
Do Trunk Muscles Affect the Lumbar Interbody Fusion Rate? : Correlation of Trunk Muscle Cross Sectional Area and Fusion Rates after Posterior Lumbar Interbody Fusion Using Stand-Alone Cage
}

\author{
Man Kyu Choi, M.D., ${ }^{1}$ Sung Bum Kim, M.D., Ph.D., ${ }^{2}$ Bong Jin Park, M.D., Ph.D., ${ }^{2}$ Chang Kyu Park, M.D., ${ }^{2}$ Sung Min Kim, M.D., Ph.D. ${ }^{3}$ \\ Department of Medicine, ${ }^{1}$ Graduate School, Kyung Hee University, Seoul, Korea \\ Department of Neurosurgery, ${ }^{2}$ Kyung Hee University Hospital, Kyung Hee University School of Medicine, Seoul, Korea \\ Department of Neurosurgery, ${ }^{3}$ Kyung Hee University Hospital at Gangdong, Kyung Hee University School of Medicine, Seoul, Korea
}

\begin{abstract}
Objective : Although trunk muscles in the lumbar spine preserve spinal stability and motility, little is known about the relationship between trunk muscles and spinal fusion rate. The aim of the present study is to evaluate the correlation between trunk muscles cross sectional area (MCSA) and fusion rate after posterior lumbar interbody fusion (PLIF) using stand-alone cages.

Methods : A total of 89 adult patients with degenerative lumbar disease who were performed PLIF using stand-alone cages at $L 4-5$ were included in this study. The cross-sectional area of the psoas major (PS), erector spinae (ES), and multifidus (MF) muscles were quantitatively evaluated by preoperative lumbar magnetic resonance imaging at the L3-4, L4-5, and L5-S1 segments, and bone union was evaluated by dynamic lumbar X-rays.

Results : Of the 89 patients, 68 had bone union and 21 did not. The MCSAs at all segments in both groups were significantly different ( $p<0.05)$ for the PS muscle, those at L3-4 and L4-5 segments between groups were significantly different $(p=0.048,0.021)$ for the ES and MF muscles. In the multivariate analysis, differences in the PS MCSA at the L4-5 and L5-S1 segments remained significant $(p=0.048,0.043$ and odds ratio=1.098, 1.169). In comparison analysis between male and female patients, most MCSAs of male patients were larger than female's. Fusion rates of male patients (80.7\%) were higher than female's (68.8\%), too.
\end{abstract}

Conclusion : For PLIF surgery, PS muscle function appears to be an important factor for bone union and preventing back muscle injury is essential for better fusion rate.

Key Words : Trunk muscle · Fusion rates · Cross-sectional area · Posterior lumbar interbody fusion.

\section{INTRODUCTION}

Lumbar interbody fusion (LIF) surgery, widely used as a major surgical treatment for degenerative spinal diseases with instability ${ }^{18)}$, has been applied to diseases such as foraminal stenosis, spondylolisthesis, and spinal deformities ${ }^{18,21)}$. According to approach, the technique is classified into anterior (ALIF), posterior (PLIF), trans-psoas (DLIF), or trans-foraminal. Successful spinal fusion surgery is marked by long-term stability of the spine, so a solid union is essential. If a bone union does not occur, patients may be suffered from failure of instrumentations or worsening of preoperative clinical symptoms.

Factors that affect bone fusion are divided into two main cat- egories : systemic and local. The systemic factors consist of insulin, endocrine hormones (sex, growth, thyroid, parathyroid, calcitonin, and the like), vitamins A, C, and D, corticosteroids, anemia, non-steroidal anti-inflammatory drugs, and smoking, among others ${ }^{1)}$. Local factors include bony surface area, mechanical stability and loading, vascularity, osteoporosis, presence of a tumor, and infection, among others ${ }^{1)}$.

Generally, surgical procedures that use mechanical instrumentation and preserve the paraspinal muscle are known to improve spinal stability ${ }^{6,7)}$. Particularly in the lumbar spine, the psoas major (PS), erector spinae (ES), and multifidus (MF) muscles are important for segmental stability and stiffness ${ }^{8)}$. Some reports discussed the relevance of atrophic, degenerative

- Received : August 5, $2015 \cdot$ Revised : October 6, $2015 \cdot$ Accepted : October 14, 2015

- Address for reprints : Sung Bum Kim, M.D., Ph.D.

Department of Neurosurgery, Kyung Hee University Hospital, Kyung Hee University School of Medicine, 23 Kyungheedae-ro, Dongdaemun-gu, Seoul 02447, Korea

Tel : +82-2-958-8402, Fax : +82-2-958-8380, E-mail : sungbumi7@hanmail.net

- This is an Open Access article distributed under the terms of the Creative Commons Attribution Non-Commercial License (http://creativecommons.org/licenses/by-nc/3.0) which permits unrestricted non-commercial use, distribution, and reproduction in any medium, provided the original work is properly cited. 
changes of the trunk muscles and spinal instability or neurologic deteriorations ${ }^{13,22)}$. Many comparison studies reported the outcomes of minimal invasive spinal surgery and classical muscle dissection surgery ${ }^{3,5,14)}$. But, despite considerable research on the relationship of other factors and bone union after lumbar fusion surgery, no study has investigated the association of trunk muscles and bone union rate after spinal fusion surgery. Accordingly, we retrospectively examined the relationship between trunk muscles and bone union rate after stand-alone cage PLIF without pedicle screw fixation (PSF) at the L4-5 segments. Despite PLIF without PSF is unpopular and unusual for achieving the fusion, it is possible to evaluate the pure effect of muscle strength by excluding the force of pedicle screw.

\section{MATERIALS AND METHODS}

\section{Patient population}

From January 2006 to December 2010, all adult patients, both male and female, with degenerative lumbar disease treated with PLIF surgery at the L4-5 segments with stand-alone cages were enrolled in this study. We excluded patients with :

1) history of previous spinal surgery; 2 ) osteoporosis, T-score below -2.5 ; 3 ) body mass index $<18$ or $>25$; 4) sagittal or coronal plane imbalance ( $40 \mathrm{~mm}$ or more translation of the $\mathrm{C} 7$ plumb line between post-superior limit of $\mathrm{S} 1,25 \mathrm{~mm}$ or more difference of the C7 plumb line between central sacral vertical axis); 5) bone metabolic or autoimmune diseases; 6) infection, pre- or postoperatively; 7) spinal tumor.

\section{Surgical technique}

In the operation room, all patients were in the prone position. After a midline incision, complete bone exposure and subperiostal dissection were performed until the transverse processes were exposed. Next, the lamina, spinous process, and supraspinatous and interspinous ligaments of L4 were resected.
The medial one-third of the facet joint and L4 -5 intervertebral disc were removed, and endplate preparation was performed with using a ring curette and shaver. Titanium cages packed with allogeneic and autologous bones were inserted into the intervertebral disc space to maintain normal disc space height. Before cage insertion, the remaining bone chips were placed anterior to the cages. One surgery team performed all surgeries using the same method. All patients had bed rest for 1 day, and then ambulated with lumbar-sacral-orthosis. Patients wore a back brace for 1-2 months after surgery.

\section{Radiologic evaluation}

All patients were followed with our clinic protocol, which includes dynamic plain radiography at two or three month intervals. Lumbar magnetic resonance imaging (MRI) was checked once, preoperatively. Bone union was evaluated by dynamic Xray, it was defined as 1) incorporation of grafts on both endplates; 2) presence of bridging trabecular bone across the interspace; 3) <2-degree movement on lateral flexion/extension views; and 4) absence of graft migration and subsidence ${ }^{2)}$.

Preoperative trunk muscle cross sectional area (MCSA) was measured in lumbar MRI. The measurement was performed by dividing the lumbar flexor muscle (PS) and extensor muscles (ES, MF) at L3-4, L4-5, and L5-S1 intervertebral spaces at right and left side on axial images (Fig. 1). The MCSA was made as the sum of both. To exclude fatty degeneration of trunk muscles, regions of increased signal intensity around the muscle were not involved. The measurement used an Infinitt Picture Archiving and Communication System. To increase intra- and inter-observer reliability, three independent observers made measurements using the same method, with accuracy coefficients of $0.79-0.87$.

\section{Statistical analysis}

Statistical analysis was performed using the Mann-Whitney U
Fig. 1. T2-weighted magnetic resonance images show the method for determining the muscle cross sectional area (MCSA). Sagittal image (A) shows the axial planes of the intervertebral space at the L3-4-5-S1 segments. Axial image (B) shows the MCSA as measured by the image software (Infinitt Picture Archiving and Communication System).
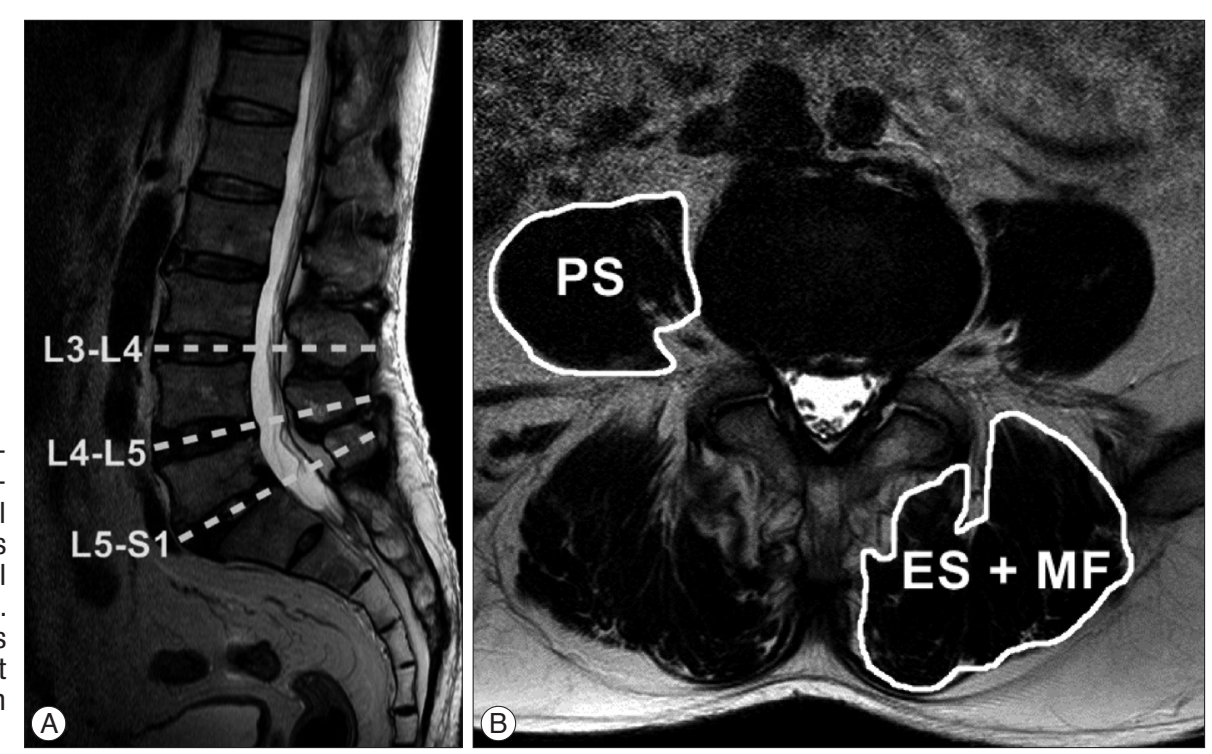
test for univariate analyses and a logistic regression test for multivariate analyses in SPSS for Windows (version 21.0; SPSS, Inc., Chicago, IL, USA). A $p$ value of $<0.05$ was considered significant.

\section{RESULTS}

Over five years, 89 patients were treated by PLIF surgery with stand-alone cages at the L4-5 segment. Patients were divided into two groups based on the presence of bone union. The union group consisted of 68 patients ( 46 men, 22 women) with a mean age of 51.7 years (range 27-73). The non-union group included 11 men and 10 women with a mean age of 58.4 years (range 27-81). The mean operative time was 14 minutes longer in the non-union than the union group, but the difference was not statistically significant $(p=0.427)$. PS MCSAs of the union group were measured as $2147.1 \pm 313.5,2591.4 \pm 361.8$, and $2592.3 \pm 341.3$ at the L3-4, L4-5, and L5-S1 segments, respec-

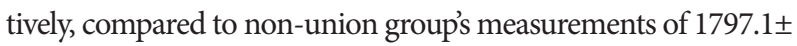
$290.3,2263.2 \pm 324.4,2181.3 \pm 311.5$ at the same segments, re- spectively. Differences between groups at all segments were significant $(p=0.018,0.041,0.014)$ (Table 1$)$. ES and MF MCSAs of the union group were measured as $4391.7 \pm 613.9,3984.3 \pm 500.1$, and 2951.3 \pm 415.6 at the L3-4, L4-5, and L5-S1 segments, compared to non-union group's measurements of $3910.1 \pm 479.0$, $3501.5 \pm 489.3$, and $2691.4 \pm 399.1$ at the same segments. Differences between groups at L3-4 and L4-5 segments were significant $(p=0.048,0.021)$ (Table 1$)$. In the multivariate logistic regression analysis, PS MCSAs at the L4-5 and L5-S1 segments remained significant $(p=0.048,0.043$ and odds ratio $=1.098,1.169$ ) (Table 2). In comparison analysis of male and female patients, expect of ES and MF MCSAs at L3-4, MCSAs of male patients were larger than female patients, significantly. Moreover, fusion rates of male patients $(80.7 \%)$ were higher than female's $(68.8 \%)$. However, there was no significant difference $(p=0.203)$ (Table 3).

\section{DISCUSSION}

Spinal surgeons focus on obtaining a solid union after spinal

Table 1. Different values in the union and non-union groups

\begin{tabular}{|c|c|c|c|}
\hline & Union group (mean $\pm \mathrm{SD})$ & Non-union group $($ mean $\pm S D)$ & $p$-value \\
\hline Mean age (yrs) & $51.7(27-73)$ & $58.4(27-81)$ & 0.196 \\
\hline \multicolumn{4}{|l|}{ Gender } \\
\hline Male & 46 & 11 & \\
\hline Female & 22 & 10 & \\
\hline Mean follow-up (months) & $20.4 \pm 6.9$ & $23.1 \pm 7.8$ & \\
\hline Operative time (min) & $176.3 \pm 29.9$ & $189.4 \pm 47.1$ & 0.427 \\
\hline \multicolumn{4}{|l|}{ PS MCSA (mm²) } \\
\hline L3-4 & $2147.1 \pm 313.5$ & $1797.1 \pm 290.3$ & $0.018^{*}$ \\
\hline $\mathrm{L} 4-5$ & $2591.4 \pm 361.8$ & $2263.2 \pm 324.4$ & $0.041^{*}$ \\
\hline L5-S1 & $2592.3 \pm 341.3$ & $2181.3 \pm 311.5$ & $0.014^{*}$ \\
\hline \multicolumn{4}{|l|}{$\mathrm{ES}, \mathrm{MF}$ MCSA $\left(\mathrm{mm}^{2}\right)$} \\
\hline L3-4 & $4391.7 \pm 613.9$ & $3910.1 \pm 479.0$ & $0.048^{\star}$ \\
\hline L4 -5 & $3984.3 \pm 500.1$ & $3501.5 \pm 489.3$ & $0.021^{*}$ \\
\hline L5-S1 & $2951.3 \pm 415.6$ & $2691.4 \pm 399.1$ & 0.109 \\
\hline
\end{tabular}

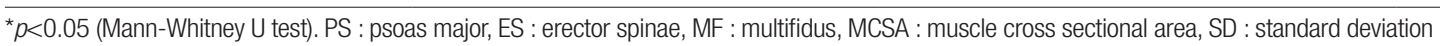

Table 2. Logistic regression analysis of multiple factors

\begin{tabular}{lcccc}
\hline & p-value & OR & \multicolumn{2}{c}{$95 \%$ CI } \\
\cline { 4 - 5 } Age & 0.145 & 0.942 & Lower limit & Upper limit \\
Gender & 0.173 & 1.006 & 0.856 & 1.030 \\
Operative time & 0.306 & 0.992 & 0.998 & 1.014 \\
Psoas major MCSA & & & 0.945 & 1.039 \\
$\quad$ L3-4 & 0.741 & 1.068 & 0.981 & 1.155 \\
L4-5 & $0.048^{*}$ & 1.098 & 0.998 & 1.198 \\
L5-S1 & $0.021^{*}$ & 1.169 & 1.002 & 1.335 \\
ES, MF MCSA & & & 0.991 & 1.041 \\
$\quad$ L3-4 & 0.849 & 1.016 & 0.982 & 1.076 \\
L4-5 & 0.182 & 1.029 & 0.990 & 1.026 \\
L5-S1 & 0.154 & 1.008 & & \\
\hline
\end{tabular}

${ }^{\star} p<0.05$. PS : psoas major, ES : erector spinae, MF : multifidus, MCSA : muscle cross sectional area, OR : odds ratio, $\mathrm{Cl}$ : confidence interval 
Table 3. Different MCSAs between male and female patients

\begin{tabular}{|c|c|c|c|}
\hline & Male $(\mathrm{n}=57)($ mean $\pm \mathrm{SD})$ & Female $(n=32)($ mean $\pm S D)$ & $p$-value \\
\hline \multicolumn{4}{|l|}{ PS MCSA } \\
\hline L3-4 & $2041.2 \pm 514.9$ & $1619.4 \pm 315.4$ & $0.009^{*}$ \\
\hline $\mathrm{L} 4-5$ & $2531.6 \pm 415.2$ & $2108.5 \pm 519.0$ & $0.032^{*}$ \\
\hline $\mathrm{L} 5-\mathrm{S} 1$ & $2603.4 \pm 419.1$ & $2206.7 \pm 451.0$ & $0.045^{\star}$ \\
\hline \multicolumn{4}{|c|}{ ES, MF MCSA } \\
\hline L3-4 & $4219.7 \pm 719.4$ & $3800.1 \pm 672.0$ & 0.058 \\
\hline L4-5 & $3959.2 \pm 627.8$ & $3511.8 \pm 510.2$ & $0.041^{*}$ \\
\hline L5-S1 & $3001.3 \pm 560.3$ & $2640.9 \pm 489.0$ & $0.011^{\star}$ \\
\hline Fusion rates & $80.7 \%(46 / 57)$ & $68.8 \%(22 / 32)$ & 0.203 \\
\hline
\end{tabular}

Fusion rate (chi-square test). ${ }^{*} p<0.05$. PS : psoas major, ES : erector spinae, MF : multifidus, MCSA : muscle cross sectional area, SD : standard deviation

fusion surgery. Non-union status can lead to recurrence of neurologic symptoms, failure of instrumentation, imbalance in coronal and sagittal plane alignment, and ultimately, reoperation and poor surgical outcomes. Thus, satisfactory decompression of neural components and successful bone union are the most important factors in the spinal surgical field. Multiple factors affect lumbar interbody fusion; of those, trunk muscles are important because they preserve overall spinal alignment and vertebral column motility and stability ${ }^{6,13,22)}$.

Anatomically ${ }^{17)}$, the PS muscle originates from the transverse process of T12-L5 and the lateral aspects of the discs between them, and inserts to the lesser trochanter of the femur. The muscle functions for 1) flexion and lateral bending in the lumbar joints and 2) flexion and rotation of the femur. Richardson et al. ${ }^{19)}$ suggest a cylinder model to explain the relationship between lumbar stability and trunk muscles. According to this model, the PS connects the pelvic floor and diaphragm, maintaining the lumbar cylinder model's stability. McGill ${ }^{16)}$ compared the lumbar spine and paraspinal muscles to a fishing rod and line, in that reducing trunk muscle tension allows the spinal segment to buckle and spinal injury to occur. The ES arises from the anterior surface of a broad and thick tendon, and then the muscular fiber splits into three columns : spinalis, longissimus, iliocostalis. The muscle is attached to the medial crest of the sacrum, the spinous process of the lumbar and the 11th and 12th thoracic vertebrae and the supraspinous ligament, the back part of the inner lip of the iliac crests, and to the lateral crests of the sacrum, where it blends with the sacrotuberous and posterior sacroiliac ligaments ${ }^{4,8)}$. The ES functions to straighten the back and provides for side-to-side rotation. The MF muscle consists of a number of fleshy and tendinous fasciculi that fill up the groove on either side of the spinous processes of the vertebrae, from the sacrum to the axis ${ }^{8)}$. Deep in the spine, the muscle spans three joint segments and works to stabilize the joints at each segmental level ${ }^{10)}$. The resulting stiffness and stability make each vertebra work more effectively and reduce degeneration of the joint structures ${ }^{8,10)}$.

In the present study, we quantitatively evaluated trunk muscle strength by measuring the cross-sectional area, which is proportional to muscular strength ${ }^{15)}$. Due to the limitations of a retrospective study design, other factors that impact muscle strength such as histologic type and degenerative changes were not assessed. So, to more precisely evaluate the correlation of fusion rate and MCSAs, we sought to exclude a number of factors affecting lumbar fusion. First, because pedicle screw fixation is stronger than other muscles in lumbar stability, we only included cases with stand-alone cages. Second, we excluded cases with sagittal or coronal imbalance and under- or overweight because axial compressive loading is affected by sagittal and coronal plane alignment and weight. Third, since osteoporosis is correlated with cage subsidence, we did not include cases with a T-score below -2.5. Fourth, we excluded cases with tumor or infection, because local microcirculation and structure deformation negatively affect fusion. Fifth, because MCSA may be different by sex, we separately analyzed male and female patients.

In our series, we found that most MCSAs in union group were larger than ones in non-union group. In addition, MCSAs of male patients were larger than those of female patients and the fusion rate of male patients were higher than those of female patients. Since the muscle volumes of men are larger than women and the paraspinal muscle affects the spinal stability, these results did not differ greatly from the common senses. However, in multivariate analysis, only PS MCSAs were correlated to spinal fusion rate. It means that the preoperative ES and MF MCSAs could not have a significant effect on the bone union. Then, why did the PS MCSA has a significant impact on spinal fusion rate than ES and MF MCSAs? We attribute this finding to two factors : PS's characteristic function and back muscle damage caused by a posterior surgical approach. First, in normal lumbar lordosis curvature, PS muscle strength is correlated with axial compression force (Fig. 2). If the PS muscle has a vector that forms an angle of $\theta$ with the sagittal vertical axis, the formula is defined; axial compression force $\alpha$ Psoas muscle strength $\times \operatorname{Cos} \theta$. The value of $\theta$ varies for each individual, but PS muscle strength and axial compression force have a clear proportional relationship. So, the PS muscle functions to increase axial compression force, which can be considered helpful for lumbar interbody fusion. While not in the same model, Janevic et al. ${ }^{12)}$ suggested that PS muscle increases the spinal stiffness by generating com- 


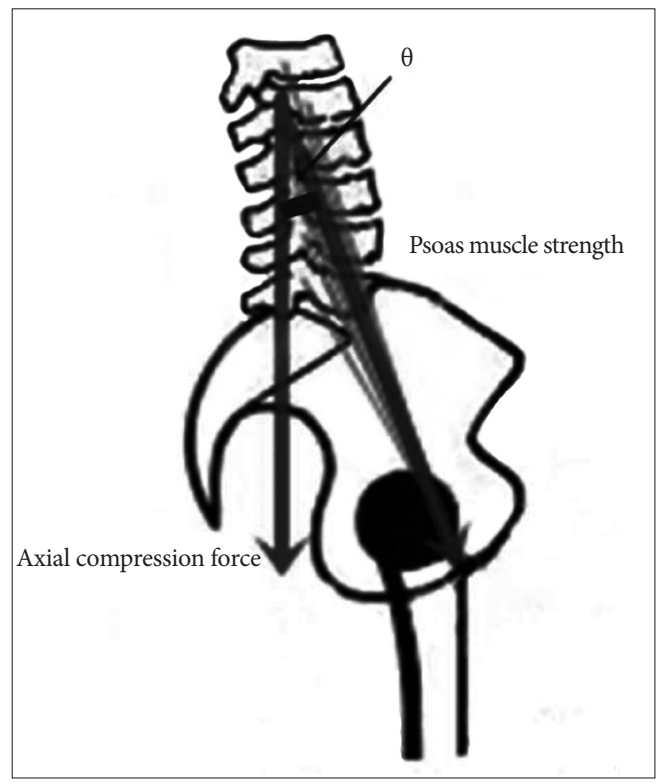

Fig. 2. The relationship between psoas muscle strength and axial compression force. The psoas muscle has a vector which forms an angle of $\theta$ with the sagittal vertical axis, establishing a proportional relationship.

pressive force through a biomechanical experiment.

Second, after lumbar fusion surgery by the classic posterior approach, the ES and MF muscles may undergo atrophy because of muscle stripping, retraction, and thermal injuries. In addition, injury to the arterial supply to muscles and dorsal rami of the posterior branches exaggerates atrophic changes ${ }^{11,20)}$. Once muscle injuries occur, it is not easy to recover initial function and strength. Hayashi et al." reported on experimental studies of denervated muscle atrophy following posterior rami of the lumbar spinal nerve in cats. In their denervation models, recovery rates in the two-level injury group did not reach $80 \%$ even after 24 weeks. Recovery time was even longer if mechanical muscle injuries also occurred. Overall, back muscles injured by the posterior lumbar surgery itself are unable to perform important roles in the bone fusion process because of their reduced strength.

The current study has some limitations. First, other surgical approaches were not compared, such as ALIF, DLIF, which is necessary to evaluate exact muscle functions (e.g., injured PS muscle and normal back muscle, such as DLIF). Second, we could not measure postoperative MCSA. If postoperative changes of trunk MCSAs had been checked, our findings would be clearer. Third, sample size was too small to draw clarified conclusions. Despite these limitations, we presume that the present study is the first report correlating trunk muscle and fusion rates by posterior lumbar fusion surgery. The PS muscle represents an important factor in preserving stability and enhancing axial compression force, which are crucial for lumbar fusion after PLIF surgery.

\section{CONCLUSION}

PS MCSAs were more correlated with spinal fusion rate after
PLIF surgery than ES and MF MCSAs. This study suggests that if preoperative trunk muscles are thin and atrophic, the fusion procedure should be done more meticulously than if they are not. In addition, prevention of back muscle injuries caused by surgical manipulation and retraction could contribute to better fusion rates.

\section{References}

1. Boden SD, Sumner DR : Biologic factors affecting spinal fusion and bone regeneration. Spine (Phila Pa 1976) 20 (24 Suppl) : 102S-112S, 1995

2. Burkus JK, Foley K, Haid RW, LeHuec JC : Surgical Interbody Research Group--radiographic assessment of interbody fusion devices : fusion criteria for anterior lumbar interbody surgery. Neurosurg Focus 10 : E11, 2001

3. Cheung NK, Ferch RD, Ghahreman A, Bogduk N : Long-term followup of minimal-access and open posterior lumbar interbody fusion for spondylolisthesis. Neurosurgery 72 : 443-450; discussion 450-451, 2013

4. Daggfeldt K, Huang QM, Thorstensson A : The visible human anatomy of the lumbar erector spinae. Spine (Phila Pa 1976) 25 : 2719-2725, 2000

5. Fan S, Hu Z, Zhao F, Zhao X, Huang Y, Fang X : Multifidus muscle changes and clinical effects of one-level posterior lumbar interbody fusion : minimally invasive procedure versus conventional open approach. Eur Spine J 19 : 316-324, 2010

6. Fan SW, Hu ZJ, Fang XQ, Zhao FD, Huang Y, Yu HJ : Comparison of paraspinal muscle injury in one-level lumbar posterior inter-body fusion : modified minimally invasive and traditional open approaches. Orthop Surg 2 : 194-200, 2010

7. Han YC, Liu ZQ, Wang SJ, Li LJ, Tan J : Comparison of unilateral versus bilateral pedicle screw fixation in degenerative lumbar diseases : a metaanalysis. Eur Spine J 23 : 974-984, 2014

8. Hansen L, de Zee M, Rasmussen J, Andersen TB, Wong C, Simonsen EB : Anatomy and biomechanics of the back muscles in the lumbar spine with reference to biomechanical modeling. Spine (Phila Pa 1976) $31: 1888-1899,2006$

9. Hayashi N, Tamaki T, Yamada H : Experimental study of denervated muscle atrophy following severance of posterior rami of the lumbar spinal nerves. Spine (Phila Pa 1976) 17 : 1361-1367, 1992

10. Hebert JJ, Kjaer P, Fritz JM, Walker BF : The relationship of lumbar multifidus muscle morphology to previous, current, and future low back pain : a 9-year population-based prospective cohort study. Spine (Phila Pa 1976) 39: 1417-1425, 2014

11. Hu Y, Leung HB, Lu WW, Luk KD : Histologic and electrophysiological changes of the paraspinal muscle after spinal fusion : an experimental study. Spine (Phila Pa 1976) 33 : 1418-1422, 2008

12. Janevic J, Ashton-Miller JA, Schultz AB : Large compressive preloads decrease lumbar motion segment flexibility. J Orthop Res 9: 228-236, 1991

13. Kaigle AM, Holm SH, Hansson TH : Experimental instability in the lumbar spine. Spine (Phila Pa 1976) 20 : 421-430, 1995

14. Lee KH, Yue WM, Yeo W, Soeharno H, Tan SB : Clinical and radiological outcomes of open versus minimally invasive transforaminal lumbar interbody fusion. Eur Spine J 21 : 2265-2270, 2012

15. Masuda K, Kikuhara N, Takahashi H, Yamanaka K : The relationship between muscle cross-sectional area and strength in various isokinetic movements among soccer players. J Sports Sci 21 : 851-858, 2003

16. McGill SM : Linking latest knowledge of injury mechanisms and spine function to the prevention of low back disorders. J Electromyogr Kinesiol $14: 43-47,2004$

17. Penning L : Psoas muscle and lumbar spine stability : a concept uniting existing controversies. Critical review and hypothesis. Eur Spine J 9 : $577-585,2000$ 
18. Resnick DK, Choudhri TF, Dailey AT, Groff MW, Khoo L, Matz PG, et al. : Guidelines for the performance of fusion procedures for degenerative disease of the lumbar spine. Part 7 : intractable low-back pain without stenosis or spondylolisthesis. J Neurosurg Spine 2 : 670-672, 2005

19. Richardson C, Jull G, Hodges P, Hides J : Therapeutic exercise for spinal segmental stabilization in low back pain : scientific basis and clinical approach. Edinburgh, London : Churchill Livingstone, 1998

20. Suwa H, Hanakita J, Ohshita N, Gotoh K, Matsuoka N, Morizane A : Postoperative changes in paraspinal muscle thickness after various lum- bar back surgery procedures. Neurol Med Chir (Tokyo) 40 : 151-154; discussion 154-155, 2000

21. Talia AJ, Wong ML, Lau HC, Kaye AH : Comparison of the different surgical approaches for lumbar interbody fusion. J Clin Neurosci 22 : 243-251, 2015

22. Teichtahl AJ, Urquhart DM, Wang Y, Wluka AE, Wijethilake P, O'Sullivan R, et al. : Fat infiltration of paraspinal muscles is associated with low back pain, disability, and structural abnormalities in community-based adults. Spine J 15 : 1593-1601, 2015 